\title{
Vasi da miele in Etruria. Confronti archeologici ed etnografici per le olle stamnoidi 'a colletto'
}

\section{Honey pots in Etruria. Archaeological and ethnographical comparisons for the stamnoid jars 'a colletto'}

\author{
Paolo Persano ${ }^{2}$ \\ Scuola Normale Superiore, Pisa, Italia
}

\section{RIASSUNTO}

L'articolo esamina le olle stamnoidi a colletto, vasi realizzati in Etruria in età ellenistica. La presenza del colletto sulla spalla, uno spazio vuoto delimitato da un cordone plastico, è un elemento che accomuna questa forma vascolare ai potes meleiros iberici e melopitari greci. Come questi, anche le olle stamnoidi possono quindi essere considerate specifici contenitori per lo stoccaggio di miele.

\section{RESUMEN}

El presente artículo analiza las ollas con forma de stamnos a colletto, vasos contenedores realizados en Etruria durante la época helenística. Estos vasos se caracterizan por tener un canal circular en el hombro, por eso es posible asociar esta forma cerámica con la de los potes meleiros ibéricos y con la de los melopitari grecos. Por eso, las ollas tienen que considerarse específicos contenedores para conservar la miel.

\section{SUMMARY}

This paper discusses stamnoid jars a colletto. These vases, made in Etruria during the Hellenistic period, are characterised by a peculiar rib that creates an empty channel around the entire circumference of the vase. Similar features can also be found on Iberian potes meleiros and Greek melopitari. Like these latter two types of vases, the aforementioned Etruscan jars may also be considered as being devoted primarily to honey storage.

PAROLE CHIAVE: vasi per la conservazione del miele; $m e$ lopitari; potes meleiros; Etruria preromana.

PALABRAS CLAVE: vasos para conservar la miel; melopitari; potes meleiros; Etruria prerromana.

\footnotetext{
${ }^{1}$ Per aver discusso con me, in anni più o meno recenti, questo studio sono particolarmente grato a D. Gandolfi (Istituto Internazionale di Studi Liguri), a L. Gambaro (Soprintendenza Archeologia della Liguria) e ad A. Ciacci (Università di Siena). Ringrazio Francesco Giancane, Andrea Masi, Cristina Nervi e Alessia Astesiano per i consigli durante la redazione del testo. 2 paolo.persano@sns.it
}

KEY WORDS: honey storage jars; melopitari; potes meleiros; pre-Roman Etruria.

\section{INTRODUZIONE}

L'archeologia del miele è stata oggetto in anni recenti di numerosi contributi che hanno messo a fuoco i differenti aspetti del ciclo produttivo di questa sostanza, troppo a lungo trascurata dalla critica $\operatorname{archeologica}^{3}$. Il dibattito sulla produzione del miele e sulla documentazione materiale disponibile per ricostruirla, dall'impianto delle arnie alle modalità del consumo, si è così arricchito negli ultimi anni di dati a integrazione di quanto attestato dalle fonti letterarie (Morel 1877; Lafaye 1904 e Bortolin 2008 appendice I). Questo nuovo quadro rende giustizia al ruolo della sostanza anche al di fuori del suo uso principale, nell'alimentazione antica per la dolcificazione e la conservazione dei cibi (André 1981: 89, 143, 186-189). Non bisogna poi trascurare le funzioni secondarie del miele: era necessario in ambito medico come antisettico, cicatrizzante ed eccipiente; nella produzione artigianale veniva usato come fissativo della porpora e un suo sottoprodotto, la cera, aveva impieghi che andavano ben oltre l'illuminazione ${ }^{4}$.

\footnotetext{
${ }^{3}$ Per una panoramica sugli studi sul miele e sulle tracce archeologiche dell'apicoltura antica: Crane 1983; Balandier 2004; Bortolin 2008 (con ampio quadro d'insieme); Bortolin 2011; Bormetti 2014; D’Agata e De Angelis 2014; Morín de Pablos e de Almeida 2014 (con dati aggiornati sulla Penisola Iberica).

${ }^{4}$ Impieghi nella medicina: Nielsen 1993; Cilliers e Retief 2008; Bormetti 2014: 32-33. Fissativo della porpora: Vitr. De Arch. 7. 13. 3; Plut. Alex. 36. 2-3, 686b; Kardara 1961: 264; Bortolin 2008: 27. Cera: Regert et alii 2001; Beretta 2009; Notarstefano 2012: 25.
} 


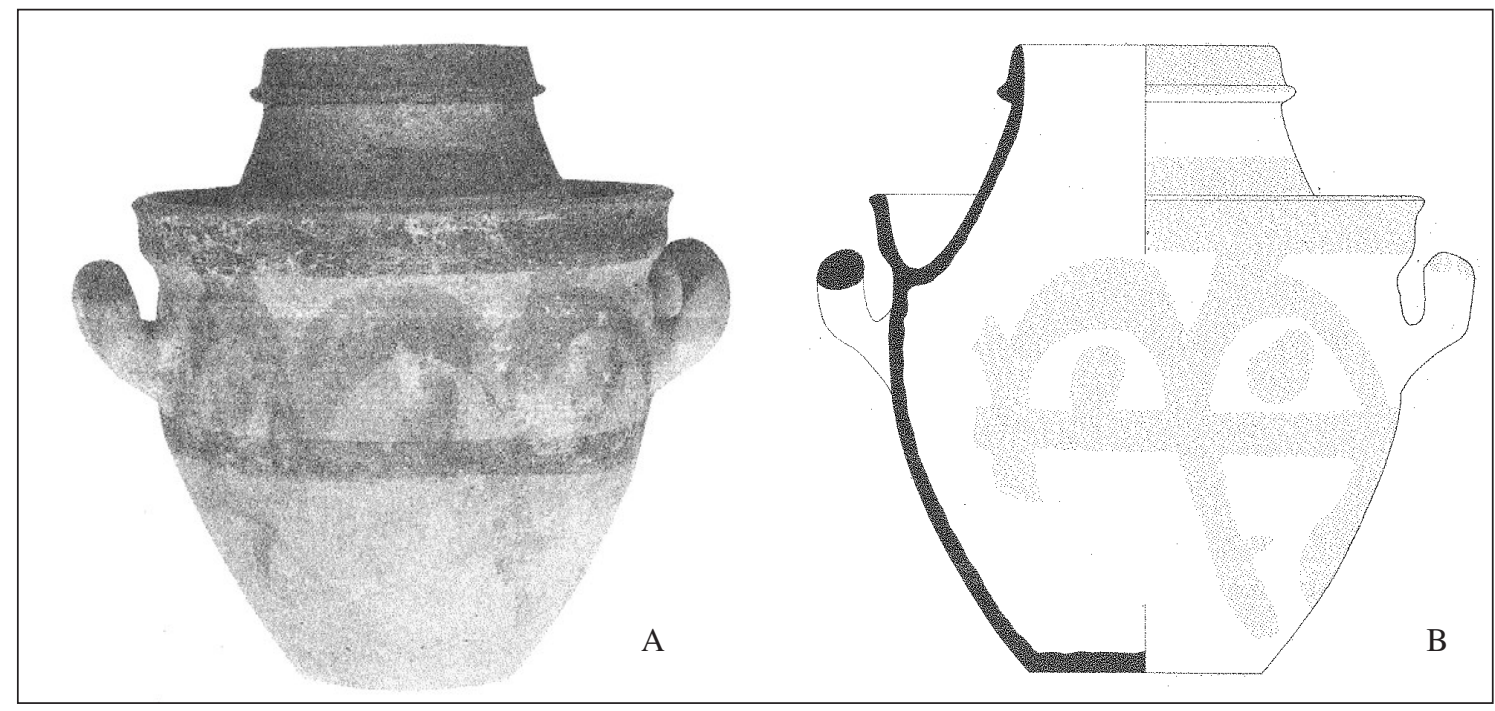

Figura 1. Olla stamnoide a colletto dalla tomba 4857 di Tarquinia a. fotografia b. disegno (da Serra Ridgway 1996: tavv. LIII e CL).

A integrazione degli studi archeologici sul miele come esito di un'attività produttiva, di frequente incentrati sull'analisi tecnologica e legati alla ricostruzione della cultura materiale antica, sono stati studiati anche gli aspetti ideologici e iconografici di questa sostanza, prova ulteriore del suo rilievo nel patrimonio culturale delle popolazioni mediterranee (Burn 1985; Cherici 1989 e, più in generale, Vázquez Hoys 1991; Giuman 2008; Giuman 2014).

Come nel resto del Mediterraneo, il miele rivestiva un ruolo significativo anche nel mondo etrusco. Qui le recenti (2013) scoperte di Forcello - Bagnolo San Vito $(\mathrm{MN})$, documentano tracce di apicoltura già nel VI secolo a.C. e la deposizione di un favo nella tomba A di Casale Marittimo (LI) attesta l'uso della sostanza nella sfera rituale (Esposito 1999: 56, 90-91).

Il presente contributo si propone di esaminare le olle stamnoidi a colletto, vasi ben noti in Etruria in età ellenistica, confrontandole con recipienti usati per lo stoccaggio di miele. Solitamente considerate cinerari, per queste olle è stata ipotizzata 'intuitivamente' una destinazione funzionale come contenitori per sostanze viscose, quale appunto il miele (Shepherd 1992: 161; Bormetti 2014: 35-36). Proprio al fine di comprenderne la funzionalità si vuole qui porre particolare attenzione ai confronti archeologici ed etnografici con vasi morfologicamente affini, cui si è accennato di recente, ma che meritano una più ampia discussione ${ }^{5}$. In questa sede si provvederà pertanto $\mathrm{a}$ : descrivere

${ }^{5}$ Jolivet 2010: 16-17, con il riferimento alle produzioni greche di età contemporanea; Rui Morais (2014a: 96; 2014b: 257) presentando brevemente il vaso da Tarcento cita la presenza di potes meleiros anche in Etruria. le olle stamnoidi a colletto etrusche illustrandone le peculiarità $(\S 2)$, confrontarle con altri vasi morfologicamente affini dalla Penisola Iberica e dalla Grecia (§3), discutere i dati archeologici e le informazioni fornite da altre fonti documentarie in riferimento ai vasi da miele (§ 4).

\section{LA FORMA VASCOLARE: MORFOLOGIA E DIFFUSIONE}

Le olle stamnoidi a colletto sono vasi di dimensioni medio-grandi realizzati in ceramica grezza, o semigrezza, di frequente verniciata (Fig. 1a-b). La forma è troncoconica, allungata, con un'imboccatura di norma ampia meno della metà del diametro del vaso sul punto di massima espansione. L'elemento diagnostico peculiare, che ha dato a questo tipo di vaso il nome correntemente utilizzato, è il colletto posto sulla spalla. Non si tratta di una decorazione applicata, ma di un vero e proprio cordone di argilla destinato a delimitare uno spazio vuoto anulare, continuo e non interrotto. L'adozione del termine colletto sembra preferibile a quella di listello, talvolta impiegata, al fine di evitare fraintendimenti con i noti 'vasi a listello' romani (Olcese 1993: 308-319). In corrispondenza dell'orlo compare di frequente un piccolo cordone applicato, da interpretarsi come alloggiamento per l'incasso di un coperchio. Anche le prese forate, sporadicamente attestate, devono essere considerate elementi destinati al fissaggio del coperchio.

La decorazione del vaso, non costante, è semplice e si limita di solito a gruppi di linee parallele o a 


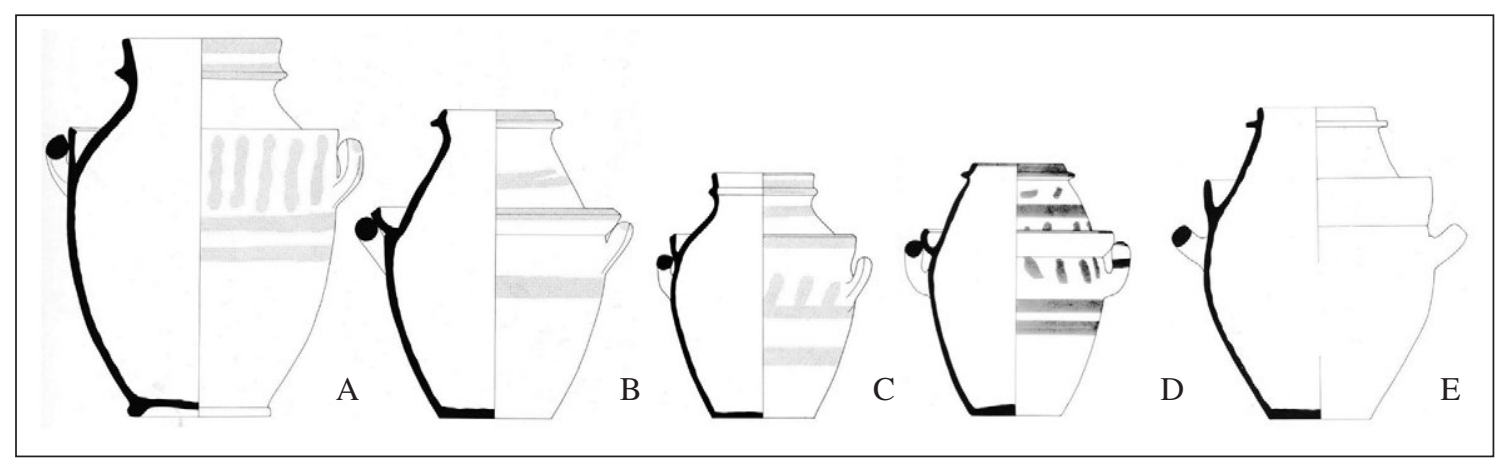

Figura 2. Olle stamnoidi a colletto da Tarquinia: a-b tomba 5070 (da Serra Ridgway 1996: tav. CLXV - 112.29-30); c. tomba 4865 (da Serra Ridgway 1996: tav. CXLIV - 69.24); d. tomba 4875 (da Serra Ridgway 1996: tav. CXLVII - 74.16); e. tomba 4826 (da Serra Ridgway 1996: tav. CCII - 163.9) (non in scala).

motivi ondulati resi con vernice di colore rosso bruno sul fondo beige-rosato della superficie del corpo ceramico. Eccezionale è invece la copertura di questi vasi con vernice nera, presente nei due soli casi, da considerarsi di produzione locale, di Norchia (cat. 37) e Tarcento (cat. 60).

Senza soffermarsi sulle variazioni morfologiche interne a questi prodotti, interessa qui discutere la forma vascolare nel suo complesso e i motivi che hanno portato all'elaborazione del modello ideale cui essa fa riferimento. Ci si limita pertanto a considerare l'olla stamnoide a colletto come una foggia che "ricopre in un dato ambiente, incarnandone la più o meno ben definita tradizione culturale, il ruolo di una certa forma funzionale; ma - a differenza da un vero e proprio tipo- non è suscettibile di una ben precisa individuazione cronologica e territoriale: abbraccia cioè svariate generazioni e cerchie regionali'" (Peroni 1998: 12).

Queste olle a colletto sono attestate per lo più in Etruria meridionale: il maggior numero di esemplari proviene dalla città di Tarquinia $(\mathrm{VT})$ e dal suo territorio (Fig. 1-3), ma l'areale di diffusione è ben più ampio, estendendosi anche in Etruria interna e settentrionale (Fig. 4).

La forma vascolare è quindi presente, seppur con una quantità limitata di esemplari, in una grande area compresa fra la provincia di Lucca, a Nord, e Minturno (LT), a Sud (Fig. 5). Per quanto riguarda la definizione dei centri di produzione di questi vasi, la documentazione edita consente con sufficiente sicurezza di isolarne in almeno quattro aree: l'agro tarquiniese, Minturno (cat. 61), il cui esemplare è un probabile scarto di fornace, il senese e l'Etruria settentrionale, da cui verrebbe anche il frammento dubbio rinvenuto a Genova (cat. 62).

Improbabile sembra invece la provenienza da Tarcento di una delle due olle a vernice nera. Il vaso,

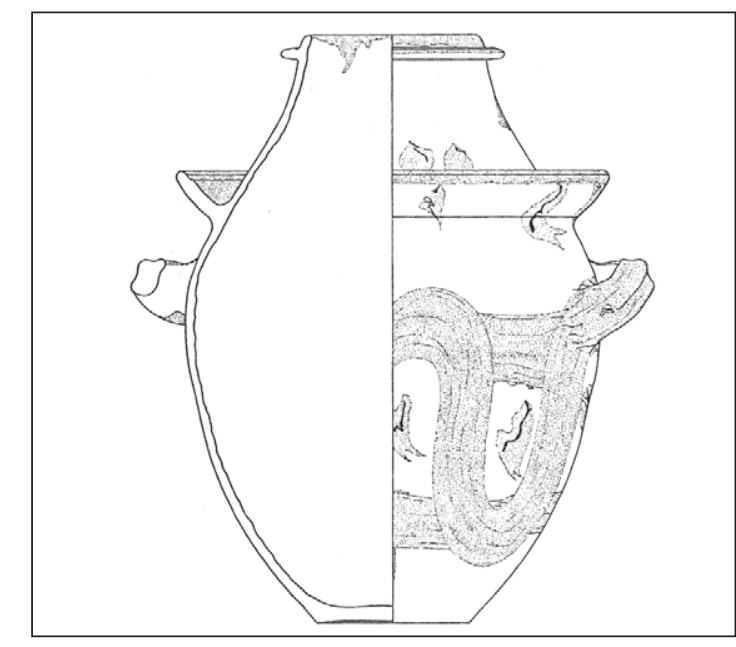

Figura 3. Olla stamnoide a colletto da Musarna (da Jolivet 2010: Fig. 4).

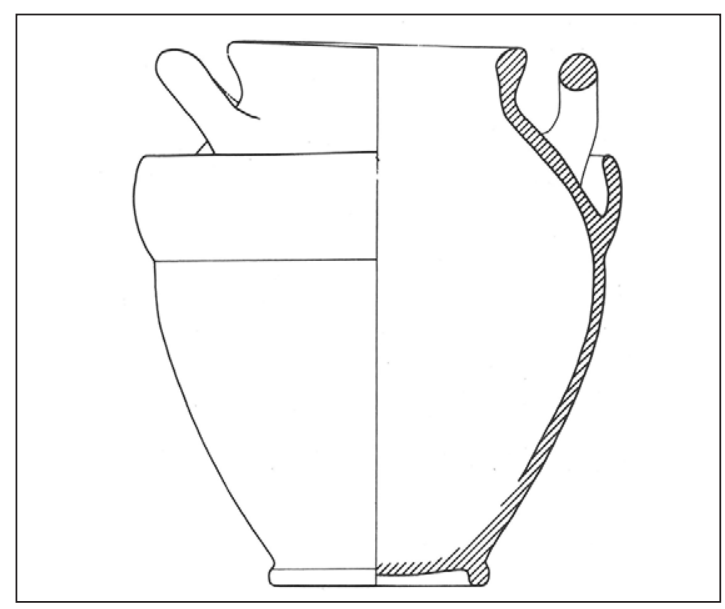

Figura 4. Olla stamnoide a colletto da Populonia (da Shepherd 1992: Fig. 27). 
ora negli Stati Uniti (cat. 60), è stato recentemente discusso da R. Morais, che ne accetta l'origine dal centro a Nord di Udine e lo connette con una produzione greca / italiota (Morais 2014a e 2014b). Tale provenienza era sembrata però non certa già all' editrice del pezzo (van Ingen 1933: 36 "said to be from Tarcento"). Prima di essere acquisito nel 1923 dall'università del Michigan, l'esemplare era nella collezione archeologica dell'università di Marburgo: vista la sua non lineare storia collezionistica e l'incompatibilità con la cultura materiale del supposto territorio di rinvenimento, tale indicazione d'origine può esser ritenuta inaffidabile.

Trattandosi di prodotti di qualità ordinaria, le olle stamnoidi a colletto erano con ogni probabilità realizzate localmente da diversi artigiani, assieme ad altri contenitori da dispensa, cui sono accomunate dall'omogeneità degli impasti e dei motivi decorativi. Sulla base della cronologia dei contesti di rinvenimento, questi vasi sembrano perdurare a lungo in età ellenistica, fra la fine del IV e il II/I secolo a.C. Talvolta una datazione più precisa non è possibile a causa della difficoltà nell'isolare le associazioni riferibili alle singole sepolture in contesti plurideposizionali (su questi aspetti Chiesa 2005).

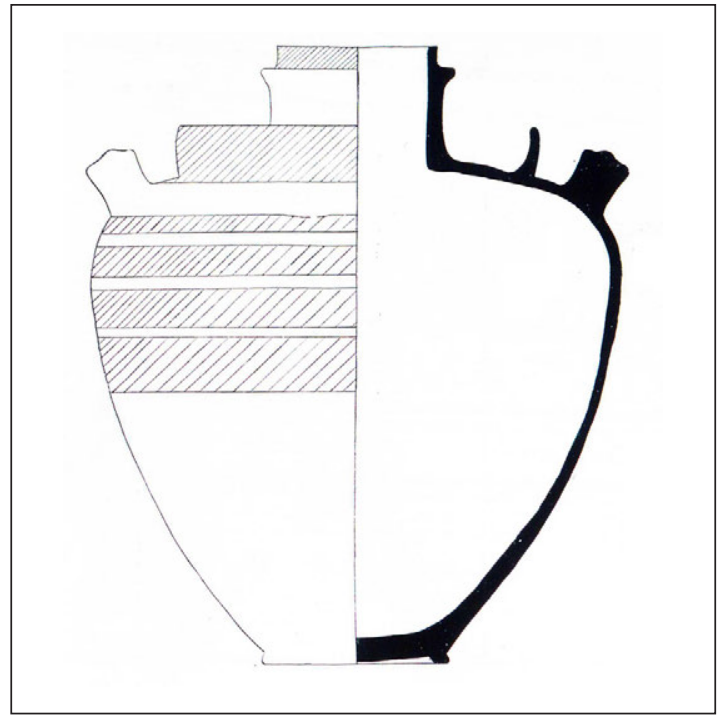

Figura 5. Olla stamnoide a colletto da Minturno (da Kirsopp Lake 1934-1935: Fig. 25).

Per una migliore discussione della documentazione archeologica riferibile a questa forma vascolare si presenta qui un elenco preliminare dei vasi, segnalandone la provenienza dall'Etruria meridionale (A), interna (B) e centro-settentrionale (C), oppure da luoghi collocati al di fuori dell'Etruria propria (D).

\section{A) ETRURia MERIDIONALE}

\section{Tarquinia}

(a questi esemplari devono essere aggiunti i vasi a colletto inediti dalla Collezione Bruschi)

1) Firenze, MAN, inv. 75910, acquisto Frangioni 1894

Martelli 1976: 47, Fig. 8; Shepherd 1992: 173, n. 1.

2) Firenze, MAN, inv. 75911, acquisto Frangioni 1894

Martelli 1976: 47, Fig. 9; Shepherd 1992: 173, n. 2.

3) tomba dei Festoni Cultrera 1920: 255-256; Shepherd 1992: 173, n. 3. 4-5) tomba delle Due Bighe Cultrera 1920: 256; Shepherd 1992: 173, nn. 4-5.

6) Reggio Emilia, Museo Civico, inv. 22555 Colonna Di Paolo e Colonna 1978: 242, n. 9; Shepherd 1992: 173, n. 6.

7) Leiden, Rijksmuseum (altezza $24,5 \mathrm{~cm}$ ) Holwerda 1936: 61, n. 888, Fig. 22; Shepherd 1992: 173, n. 7.

8) Edinburgh, Royal Scottish Museum Serra Ridgway 1986: 351, n. 72; Shepherd 1992: 173 , n. 8 .

9) Viterbo, MAN, Collezione Rossi Danielli, inv. $341 / 297$ (h. $23 \mathrm{~cm}$; diam. orlo 10,5 cm) Emiliozzi 1974: 193, tav. CXL, n. 298; Shepherd 1992: 174, n. 11 (proveniente dal territorio di Tarquinia).

10) Viterbo, MAN, Collezione Rossi Danielli, inv. $341 / 556$ (h. 29,5 cm; diam. orlo $10 \mathrm{~cm}$ )

Emiliozzi 1974: 193, tav. CXL, n. 299; Shepherd 1992: 174, n. 12 (proveniente dal territorio di Tarquinia).

11) Fondo Scataglini, tomba 4789 Serra Ridgway 1996: 124, t. 105.39 (sul contesto Chiesa 2005: 124-125).

12) Fondo Scataglini, tomba 4826 (h. $28 \mathrm{~cm}$ - Fig. 2e) Serra Ridgway 1996: 190, t. 163.9, tav. CCII (sul contesto Chiesa 2005: 126).

13) Fondo Scataglini, tomba 4835 Serra Ridgway 1996: 198, t. 169.60 (sul contesto Chiesa 2005: 127).

14) Fondo Scataglini, tomba 4836 (h. $26,5 \mathrm{~cm}$ ) Serra Ridgway 1996: 200, t. 170.24, tav. CCV (sul contesto Chiesa 2005: 153).

15) Fondo Scataglini, tomba 4857 (h. 21,8 cm; diam. orlo $9 \mathrm{~cm}$ - Fig. 1)

Serra Ridgway 1996: 102, t. 83.9, tav. CL (sul contesto Chiesa 2005: 116).

16) Fondo Scataglini, tomba 4862

Serra Ridgway 1996: 100, t. 79.6 (sul contesto Chiesa 2005: 123-124). 
17) Fondo Scataglini, tomba 4865 (h. $23 \mathrm{~cm}$ - Fig. 2c) Serra Ridgway 1996: 86, t. 69.24, tav. CXLIV (sul contesto Chiesa 2005: 141).

18) Fondo Scataglini, tomba 4870

Serra Ridgway 1996: 95, t. 76.14 (sul contesto Chiesa 2005: 123).

19) Fondo Scataglini, tomba 4875 (h. $25 \mathrm{~cm}$ - Fig. 2d) Serra Ridgway 1996: 94, t. 74.16, tav. CXLVII (sul contesto Chiesa 2005: 122-123).

20) Fondo Scataglini, tomba 4918 (h. 19,5 cm; diam. orlo $8,5 \mathrm{~cm}$ )

Serra Ridgway 1996: 70, t. 57.22, tav. CXXXV (sul contesto Chiesa 2005: 138-139).

21) Fondo Scataglini, tomba 4920

Serra Ridgway 1996: 170, t. 147.6 (sul contesto Chiesa 2005: 114).

22) Fondo Scataglini, tomba 5001

Serra Ridgway 1996: 154, t. 135.3 (sul contesto Chiesa 2005: 109).

23) Fondo Scataglini, tomba 5048

Serra Ridgway 1996: 194, t. 165.53 (sul contesto Chiesa 2005: 152).

24) Fondo Scataglini, tomba 5061

Serra Ridgway 1996: 173, t. 151.15 (sul contesto Chiesa 2005: 148-149).

25) Fondo Scataglini, tomba 5064

Serra Ridgway 1996: 66, t. 52.20 (sul contesto Chiesa 2005: 122).

26) Fondo Scataglini, tomba 5067

Serra Ridgway 1996: 150, t. 131.6 (sul contesto Chiesa 2005: 125-126).

27) Fondo Scataglini, tomba 5070 (h. 29,3 cm; diam. orlo $12 \mathrm{~cm}$ - Fig. 2)

Serra Ridgway 1996: 131, t. 112.29, tav. CLXV (sul contesto Chiesa 2005: 95-98, 144-145).

28) Fondo Scataglini, tomba 5070 (h. $23,5 \mathrm{~cm}$; diam orlo 8,8 cm - Fig. 2)

Serra Ridgway 1996: 131-132, t. 112.30, tav. CLXV.

29) Fondo Scataglini, tomba 5073

Serra Ridgway 1996: 30, t. 21.7 (sul contesto Chiesa 2005: 119).

30) tomba 5512, inv. 102607 e 102608 (h. 27,2 cm) Cavagnaro Vanoni 1996: 230-231, Fig. 77.16 (sul contesto Chiesa 2005: 53-54).

31) tomba 5580

Cavagnaro Vanoni 1986: 349, n. 15; Serra Ridgway 1996: 266 (sul contesto Chiesa 2005: 56).

Norchia

32) tomba PA 1

Colonna Di Paolo e Colonna 1978: 242-243, tavv. CCCL 1, CCCCLI; Shepherd 1992: 174, n. 13.

33) tomba PA 39
Colonna Di Paolo e Colonna 1978: 294, nn. 5253, tav. CCCLXIII; Shepherd 1992: 174, n. 14.

34) tomba PA 65

Colonna Di Paolo e Colonna 1978: 352-353, tav. CCCCIV; Shepherd 1992: 174, n. 14.

35-36) località Acqualta tomba A 14, due esemplari (h. 34 e $30 \mathrm{~cm}$ ).

Gargana 1936: 276, nn.18-19; Shepherd 1992: 174, nn. 16-17.

37) tomba in località Fosso Pile, loculo IV, inv. 112.239

Barbieri 2003: 227, Fig. 2.

Castel d'Asso

38) Toronto, Royal Ontario Museum, inv. G5 (h. 25,7 $\mathrm{cm}$; diam. orlo 10,1-10,7 cm)

Hayes 1985: 163; Shepherd 1992: 174, n. 18.

\section{Musarna}

39) cisterna 642 (Fig. 3)

Jolivet 1999: 482, Fig. 2; Jolivet 2010: 16-17.

40) tomba XX, Viterbo, MNA, Collezione Rossi Danielli (h. $15 \mathrm{~cm}$, diam. orlo $8,2 \mathrm{~cm}$ )

Emiliozzi 1974: 56-57, tav. XIX; Shepherd 1992: 174, n. 19.

Tuscania

41) tomba dei Curunas (h. $28 \mathrm{~cm}$, diam. $12 \mathrm{~cm}$ ) Moretti e Sgubini Moretti 1983: 138, n. 306, Fig. 20.1 .

Monte Romano

42) dalla 'Grotta delle Statue'

Fortini 1987: 44, n. 18.

Pyrgi

43) frammenti di anse da riferire probabilmente a olla stamnoide

Serra 1970: 525, n. 97; Shepherd 1992: 174, n. 29.

44) frammento di parete di olla con fascia aggettante (colletto?)

Serra 1970: 646, n. 7; Shepherd 1992: 174, n. 30.

\section{B) ETRURIA INTERNA}

Asciano

45) tumulo dei Marcni, già Firenze, MAN, inv. 102574 (ora Museo di Asciano)

Shepherd 1992: 174, n. 22.

Monteriggioni

46-50) cinque esemplari inediti

Shepherd 1992: 174, nn. 23-27. 


\section{Montaperti}

51) perduta, già nella Collezione Tommasi (rinvenuta nell'ottobre 1728 nelle proprietà dei Tommasi fra Dofana e Pancole - Fig. 14)

Cristofani 1979: 180, n. 15, Fig. 143/15; CIE: 42, n. 237 (per il contesto Cristofani 1979: 179-183).

Orvieto

52) Firenze, MAN, inv. 77512 , Martelli 1976: 47, Fig. 10; Shepherd 1992: 174, n. 20.

\section{Monte San Savino}

53) Firenze, MAN, acquisto Orsi 1901 Martelli 1981: 423, n. 94; Shepherd 1992: 174, n. 21.

\section{C) Etruria CENTRo-SETtentrionale}

Cosa

54) Grosseto, Museo Archeologico e d'Arte della Maremma

Mazzolai 1977: 91 (sala XVII, vetrine A-C, dalla suppellettile di una casa); Martelli 1981: 423, n. 94; Shepherd 1992: 174, n. 31.

55) Pottery Dump 3, flanged pan in ceramica da cucina Dyson 1976: 88, Fig. 28 - PD3; Shepherd 1992: 174, n. 32.

56) Pottery Dump 3, flanged pan in ceramica da cucina Dyson 1976: 88, Fig. 28 - PD4; Shepherd 1992: 174, n. 33.

\section{Populonia}

57) tomba 30/1908, Firenze, MAN, inv. 11908 (Fig. 4) Martelli 1981: 423, tav. CV.2; Romualdi 1985: 192, n. 40; Shepherd 1992: 160-162 (sul contesto Romualdi 1985: 188-193).

Ponte a Moriano (Campo della Madonna presso $S$. Geminiano di Moriano)

58) tomba (h. $28 \mathrm{~cm}$ )

Custer 1928: 29, Fig. 3 a sinistra; Shepherd 1992 : 174, n. 35 .

59) tomba (h. $28 \mathrm{~cm})$

Custer 1928: 29, Fig. 3 a destra; Shepherd 1992: 174, n. 36

\section{D) Al Di FUORI DELl'Etruria}

\section{Tarcento (?)}

60) Michigan, University inv. 2623 (h. 25,9 cm; larghezza $22,7 \mathrm{~cm}$ ) van Ingen 1933: 36, tav. XIX.15; Martelli 1981: 423, n. 94; Shepherd 1992: 174, n. 38 ; Morais 2014a: 96-97; Morais 2014b, 257.

\section{Minturno}

61) da una fornace (?) (h. ipotetica ricostruita 47,5 $\mathrm{cm}$; diam. fra 11,4 e $10,6 \mathrm{~cm}$ - Fig. 5)

Kirsopp Lake 1934-1935: 104-105, tipo 25, tavv. 15-16; Shepherd 1992: 174, n. 28.

Genova

62) dagli scavi di San Silvestro (si tratta solo di un frammento di collo e potrebbe riferirsi anche a una differente forma vascolare, l'identificazione è pertanto dubbia)

Milanese e Mannoni 1984: 143, Fig. 9.9; Shepherd 1992: 174, n. 37

Come si può evincere dall'elenco, le informazioni sui rinvenimenti, disponibili solo in alcuni casi, documentano per lo più la provenienza da tombe, dove le circostanze di conservazione hanno consentito la sopravvivenza di vasi quasi integri. Nel caso di esemplari frammentari i resti del colletto possono infatti essere attribuiti a orli di contenitori di più grandi dimensioni, come è avvenuto a Cosa (Dyson 1976: 88 - flanged pan) e come è frequente nel caso di forme vascolari insolite (cfr. Di Gennaro e Depalmas 2011: 59).

La presenza di ossa umane combuste all'interno di alcuni di questi vasi ha consentito di proporne la destinazione funzionale di cinerari. A titolo esemplificativo si possono ricordare alcuni rinvenimenti nella necropoli del Fondo Scataglini a Tarquinia o i due esemplari dalla tomba di Ponte a Moriano (cat. 58-59) e, purtroppo perduto, il vaso con epigrafe dalla Collezione Tommasi nel senese (cat. 51).

Tale destinazione, certa per un buon numero di casi, è però condivisa con altri vasi di uso domestico come i boccali e le più comuni olle globulari o ovoidali, evidentemente divenuti cinerari solo in un secondo momento (Serra Ridgway 1996: 266). Il fatto che alcune di queste olle, alla fine del loro ciclo di vita, abbiano contenuto resti umani e ci siano pertanto pervenute in corredi tombali non è di per sé prova di un'esclusiva destinazione funeraria (cfr. Shepherd 1992: 161). Tale impiego sembra pertanto dipendere dai contesti di rinvenimento piuttosto che da una specificità originaria della forma rispetto ai vasi omologhi (olle) in ceramica comune (Martelli 1976: 47; Serra Ridgway 1986: 327; Serra Ridgway 1996; Cavagnaro Vanoni 1996).

Certi sono anche i rinvenimenti in contesti domestici o comunque non funerari come a Musarna, 
Pyrgi e Cosa (cat. 39, 43-44, 54-56; Jolivet 1999: 481-482): la loro scarsità sembra dipendere da una minore visibilità nella documentazione archeologica, la cui massa critica, in Etruria più che in altre regioni, è legata allo scavo delle necropoli.

\section{I confronti: Penisola Iberica, Italia, Grecia}

Questa forma vascolare può pertanto essere descritta come un comune vaso chiuso di dimensioni medio-grandi destinato allo stoccaggio e connotato dalla presenza del colletto, attributo morfologico la cui giustificazione non dovrà essere connessa a scelte stilistiche, ma alle esigenze funzionali del vaso. Il lungo perdurare di tale specifica caratteristica formale e l'ampia diffusione geografica dei prodotti suggeriscono infatti un'interpretazione legata alla funzione del vaso, analogamente a quanto riscontrabile per altre forme specializzate (e.g. Gambaro 2006). Il confronto con esemplari provenienti da differenti aree del bacino mediterraneo, per cui è stata riconosciuta una destinazione funzionale su base etnografica, può quindi costituire un utile strumento per la comprensione di questi vasi etruschi.

Il dossier di confronto offerto dalla Penisola Iberica, di notevole importanza e per numero di attestazioni e per lunga durata cronologica, deve essere senza dubbio considerato per primo. L'attributo formale diagnostico delle olle etrusche qui discusse, il colletto, si ritrova infatti pressoché identico in un ampio insieme di vasi rinvenuti in contesti di scavo della Spagna e del Portogallo. Si tratta di contenitori destinati, come attestano confronti etnografici e analisi archeometriche, allo stoccaggio e al trasporto di miele o di analoghe sostanze semi-liquide pregiate. Lo spazio vuoto costituito dal colletto riveste un valore funzionale ben preciso: riempito d'acqua impedisce che gli insetti, le formiche in particolare, possano penetrare nel vaso rovinando le scorte alimentari contenute (Fletcher 1953: 191; Delgado 1996-1997: 150).

Questi esemplari, grazie ai confronti etnografici con la produzione artigianale locale chiamati potes meleiros, pucheiros miel, cántaros de miel, vasos meleiros o talhas meleiras, sono stati oggetto nell'ultimo ventennio di studi analitici volti a dimostrarne la persistenza nella longue durée. Tale continuità cronologica può essere spiegata esclusivamente attribuendo un valore funzionale alla morfologia del vaso che, venendo incontro a una necessità effettiva, è stata replicata per lungo tempo. Trattandosi di un prodotto utile a un determinato scopo, seppur evidentemente non indispensabile, la sua forma è sopravvissuta nella produzione tradizionale di contenitori in terracotta,

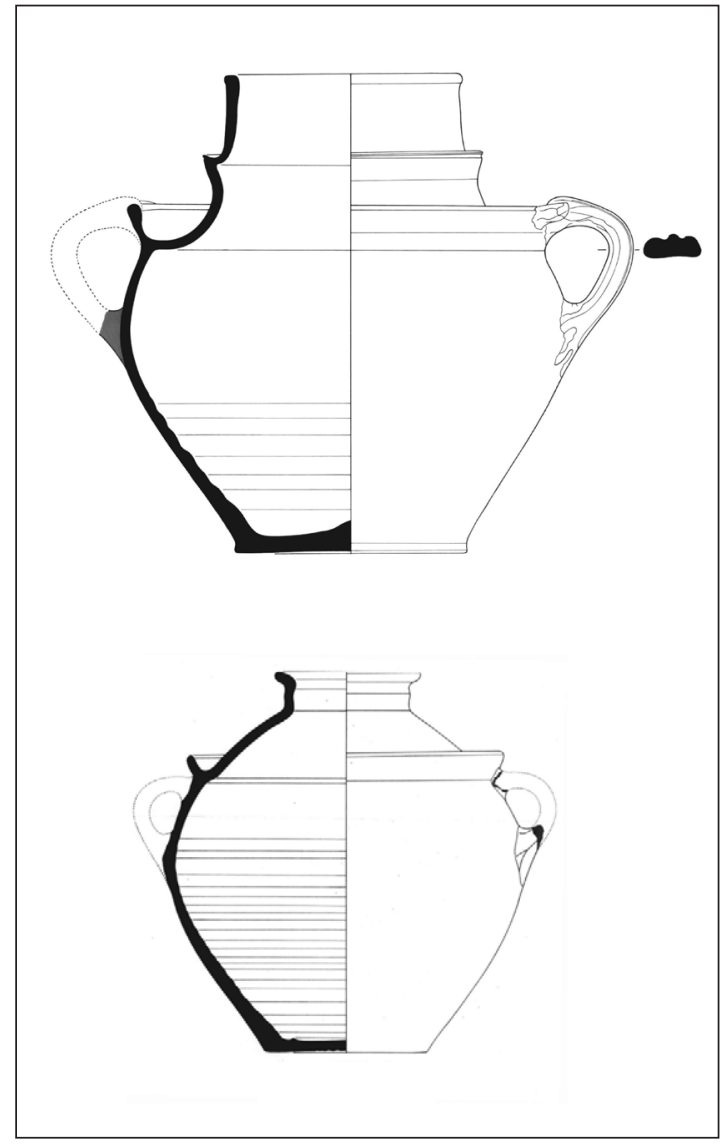

Figura 6. Potes meleiros da Bracara Augusta (da Delgado 19961997: Fig. 4.2-3).

rimanendo nel repertorio vascolare locale (Delgado 1996-1997; Morais 2006: 151-156). Fra i potes meleiros rinvenuti in depositi archeologici certi si segnalano in particolare quelli, databili all'età imperiale romana, editi da Manuela Delgado e provenienti dagli scavi nella "zona das Carvalheiras" della città di Bracara Augusta in Portogallo (Fig. 6) (Delgado 1996-1997).

La studiosa ha per prima valorizzato la straordinaria analogia fra questi vasi e le produzioni tradizionali, moderne e contemporanee, di contenitori per miele dei centri di Telhal (Castelo Blanco), Fazamões (Lamego), Felgar (Torre de Moncorvo), Bizalhães (Vila Real) (Gomes 2007: 78).

Grazie ai confronti portoghesi è stato possibile precisare la funzione di una forma vascolare dell'Età del Ferro iberica nota da tempo (Fig. 7), il cui colletto era stato già riferito ipoteticamente alla refrigerazione o all'isolamento dei cibi (Fletcher Valls 1953: 191; Mata Parreño e Bonet Rosado 1992: 127, Fig. 4 e 142, Fig. 28). Lo spostamento di questi vasi, e naturalmente dei loro contenuti, all'interno della Penisola Iberica 
fra il IV e il II secolo a.C. è poi attestato dal rinvenimento in Portogallo di un esemplare di produzione andalusa (Gomes 2007: 86). Recentemente analisi archeometriche ne hanno confermato la funzionalità, riscontrando la presenza di residui organici di miele e cera (Oliveira e Morais 2014: 40, 44-45; Oliveira et alii 2014).

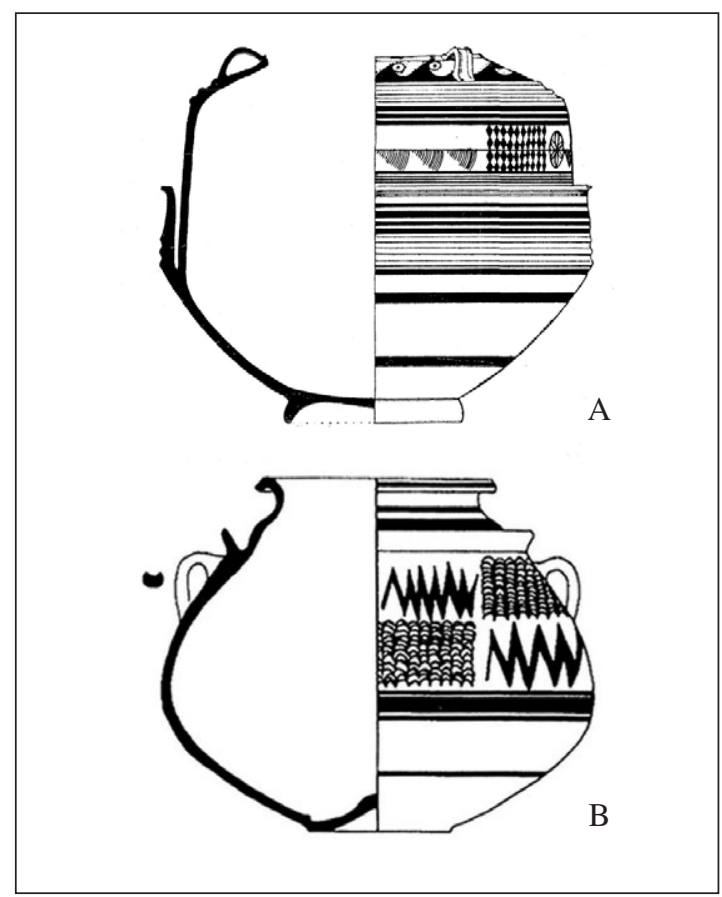

Figura 7. Potes meleiros di produzione iberica (a. Fletcher Valls 1953: Fig 2; b. da Morais 2006: Fig. 1.3).

In Italia la documentazione archeologica sui vasi a colletto differenti dalle olle etrusche si presenta invece limitata. Di notevole importanza per il presente discorso è un'olla, pubblicata recentemente, dalla Raccolta Aprosiana nel Museo Civico Archeologico Girolamo Rossi di Ventimiglia (Fig. 8). Il vaso è stato considerato dagli editori, per i confronti tipologici e per l'analisi macroscopica dell'impasto, un prodotto di età imperiale verisimilmente importato dalla Spagna (Gandolfi e Gervasini 2005: 91; Gandolfi e Mennella 2012). Rientrante totalmente nella forma vascolare dei potes meleiros iberici, l'olla si connota per la straordinaria presenza di un'iscrizione post cocturam che ne supporta l'interpretazione come contenitore destinato allo stoccaggio di miele. Il testo è stato così letto da G. Mennella Tes(ta) p(ondo libras) VIII, mel (pondo libras) VII (uncias) II (Gandolfi e Mennella 2012: 338-340).

Al di fuori della Penisola Iberica ed escludendo quest'esemplare, forme vascolari chiuse a colletto

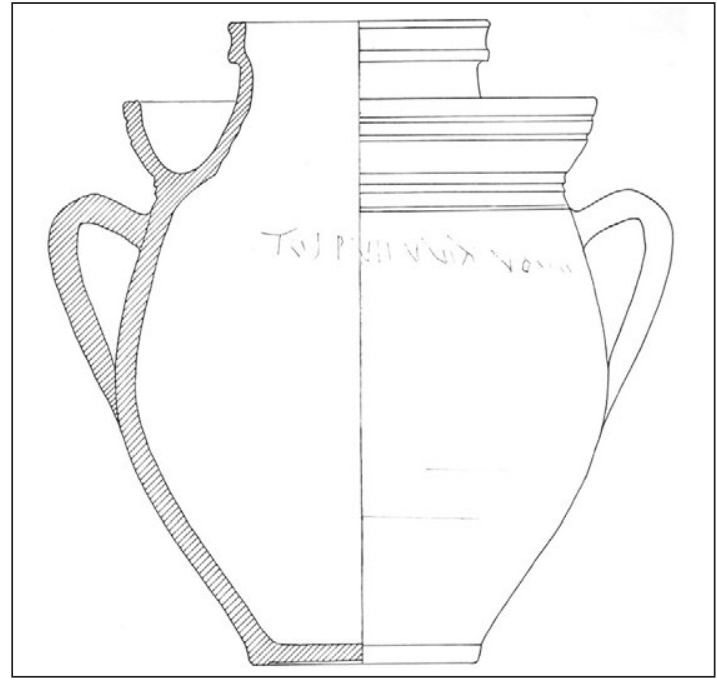

Figura 8. Ventimiglia, Museo Civico Archeologico Girolamo Rossi, olla (da Gandolfi e Mennella 2012: Fig. 1).

non sembrano comparire nell'instrumentum domesticum romano. Certo non possono essere ascritti a questa categoria né i prodotti in ceramica fine, come la piccola olla megarese dal colletto poco pronunciato (da considerarsi una sagomatura per l'appoggio del coperchio - Arena 1969: 109-111, n. 7; Puppo 1995: 74-75, T25) né una peculiare forma vascolare attestata nella Francia del Sud (Brentchaloff 1980: 105, tav. VI, n. 21; Rivet 1982: 260, forma 21; Bérato 2009: 398-399, forma 1626 - Fig. 9). In particolare caratteristiche formali (il grande diametro dell'imboccatura) e tecniche (la loro pertinenza alla ceramica culinaire modelée varoise) obbligano a ipotizzare per i prodotti francesi una differente destinazione d'uso.

L'orlo interno dei cd. Milchkocher, per la caratteristica presenza dei fori deve essere interpretato come atto a consentire il passaggio dell'aria o di liquidi: è pertanto concettualmente il contrario del colletto dei contenitori qui discussi (cfr. Ettlinger et alii 1990: 140, forma 51.1-2; Santrot e Santrot 1995: 189-192, nn. 511-512; Hayes 2008: 255-256, n. 1477).

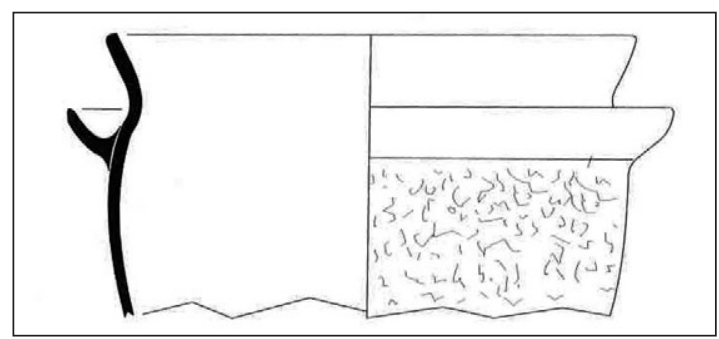

Figura 9. Francia del Sud, Ceramica modelée varoise forma Bérato 1626 (da Bérato 2009: 399, Fig. 16). 
Agli esemplari iberici prodotti fino al secolo scorso possono invece essere avvicinate con ogni probabilità anche forme vascolari contemporanee di provenienza greca, in particolare insulare, note, almeno a Creta, con il nome di melokouroupa (Figg.10-11) o melopitaro (Fig. 12).

Anche in questo caso la destinazione funzionale per lo stoccaggio di miele è attestata etnograficamente e si basa su un criterio identico a quello riscontrabile

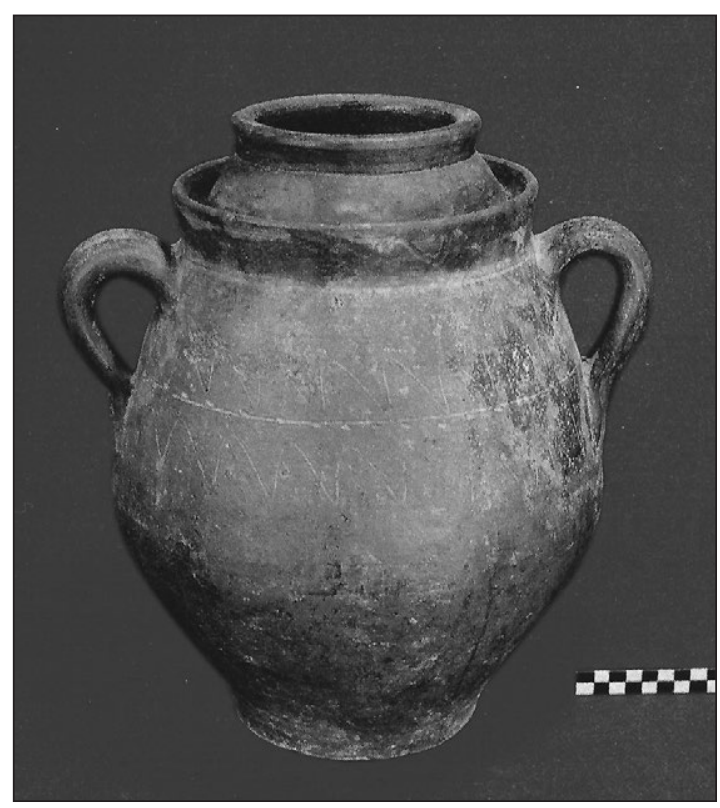

Figura 10. Contenitore per miele di età contemporanea da Creta

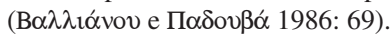

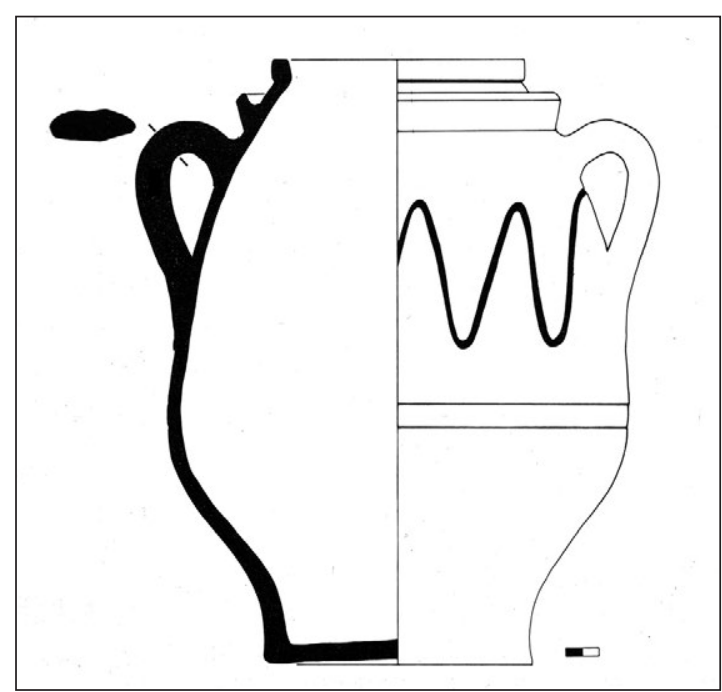

Figura 11. Contenitore per miele di età contemporanea da Creta (B $\alpha \lambda \lambda$ ıó́vov e П $\alpha \delta$ ovßó 1986: 45). nei vasi iberici; nel caso del melopitaro la capacità oscilla fra i 10 e i $200 \mathrm{~kg}$, mentre di dimensioni minori sembra essere la melokouroupa che si contraddistingue anche per un profilo più vicino a quello dei prodotti

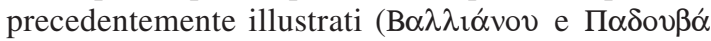
1986: 40, 45).

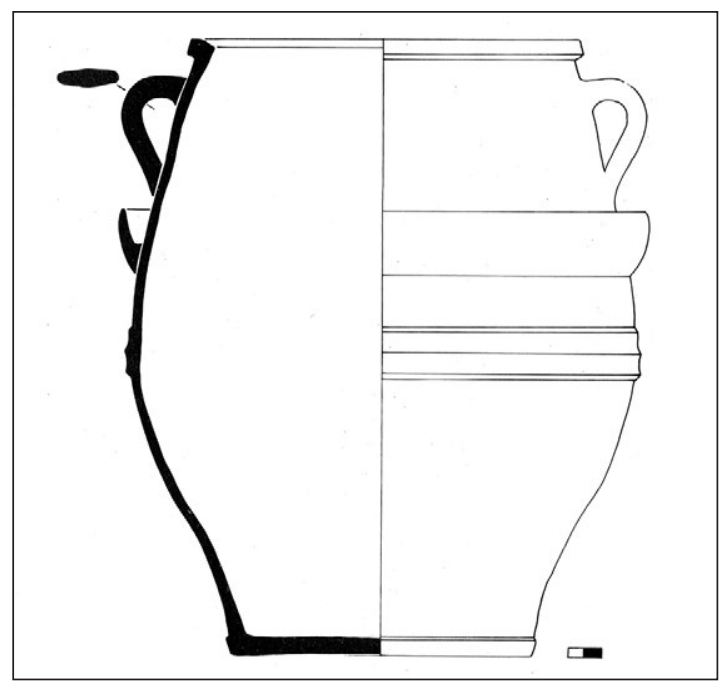

Figura 12. Contenitore per miele di età contemporanea da Creta

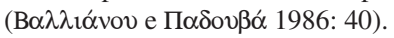

L'analogia è evidente confrontando, per esempio, le olle etrusche a colletto con gli esemplari conservati nel museo etnografico di Vori, nella pianura della Messarà a Creta (Figg. 10-11) o nel museo del folklore di Neapolis, non lontano da Aghios Nikolaos. Vasi con forma analoga sono noti anche nelle Cicladi, dove confronti etnografici ne documentano la medesima funzione (Fig. 13). Anche il vaso di provenienza cicladica al Museo di Sèvres (SN 459, Massoul 1934: 13, IIBb1, tav. 6.1,), per cui è stata proposta l'identificazione con un'olla stamnoide a colletto etrusca (Di Paolo Colonna e Colonna 1978: 243), si può inserire in questo insieme di contenitori da miele. Una conformazione avvicinabile del colletto sembra comparire anche nel repertorio levantino di età medievale (Gabrieli 2009: 71, Fig. 6:4.4). Le stringenti analogie morfologiche fra i vasi a colletto iberici e greci, di cui è ricostruibile con certezza la funzione preferenziale, e le olle etrusche consentono pertanto di ipotizzare anche per quest'ultime il medesimo impiego di contenitori per il miele.

Nonostante la straordinaria importanza del miele fra i prodotti del mondo greco, documentata da fonti letterarie non solo antiche, vasi a colletto non sembrano essere conservati nei depositi archeologici, dove peraltro abbondano resti riferibili alla pratica 


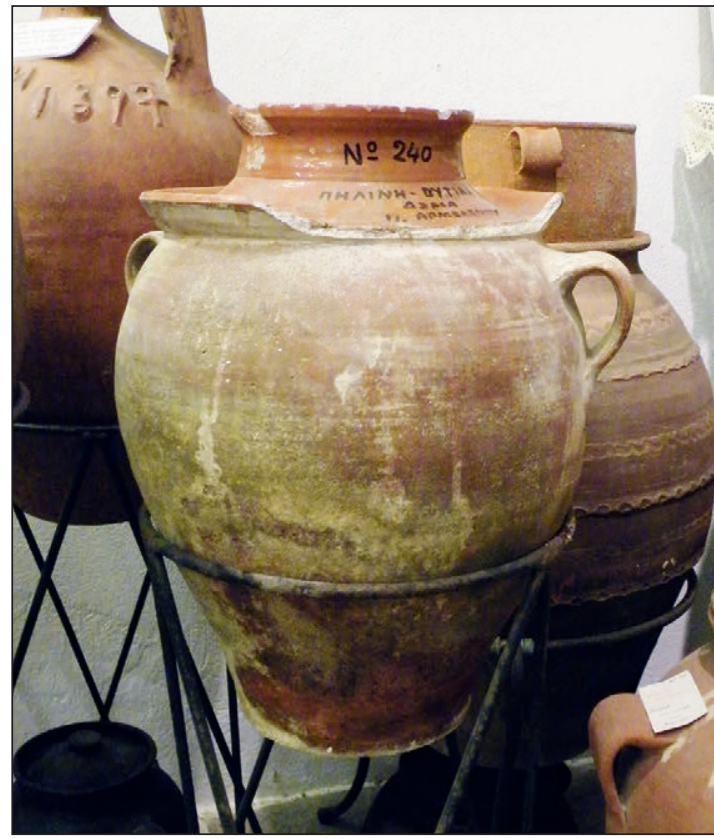

Figura 13. Contenitore per miele di età contemporanea da Sifno, museo etnografico di Apollonia (foto Paolo Persano).

intensiva dell'apicoltura, in particolare arnie. Nella Grecia classica ed ellenistica il miele doveva quindi essere stoccato in contenitori differenti. Per la conservazione di alimenti è per esempio ben nota, ad Atene e Delo, una forma di olla stamnoide con un listello appiattito vicino all'orlo (Rotroff 2006: 94, figg. 24-26; Peignard-Giros 2012: 248-249, Fig. 3).

Si tenga comunque presente che, lo studio del ciclo di produzione del miele si è concentrato in particolare sul riconoscimento e sulla definizione delle arnie. La varietà tipologica di questi oggetti è stata ampiamente analizzata, sia in riferimento al mondo iberico sia a quello greco, dove sono attestate arnie verticali e orizzontali ${ }^{6}$. Bisogna comunque ricordare che, nonostante le fonti affermino esplicitamente che le migliori arnie siano in corteccia, ferula e vimini (Plin., HN 21. 80; Columella, Rust. 9. 6. 1-2; Varro, Rust. 3. 16. 15), noi conosciamo questi oggetti solo quando realizzati in terracotta o pietra (Bormetti 2014: 16-17).

Degni di menzione, oltre ai potes meleiros, sono anche altri vasi di produzione iberica, i kalathoi sombreros de copa, da tempo riferiti allo stoccaggio di miele (Del Chiaro 1973: 65). La destinazione fun-

${ }^{6}$ Penisola Iberica: Morais 2006: 156-157. Grecia: Jones et alii 1973: 443-452; Lawall et alii 2001: 176-177; AndersonStojanović e Jones 2002; Rotroff 2006: 124-131 (con ampia

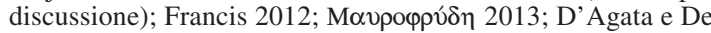
Angelis 2014. zionale dei sombreros de copa, ben noti e documentati anche in Italia, è però tutt'altro che chiara: l'orlo sembra inadatto ad accogliere una solida chiusura e altrettanto verisimili sono le ipotesi che questi vasi contenessero prodotti sotto sale e frutta $\mathrm{secca}^{7}$. Bisogna però ricordare come analisi archeometriche su alcuni esemplari, rinvenuti nel sito del Torrelló del Boverot de Almazora (Castellón), abbiano identificato residui di un preparato di miele e frutti carnosi o mosto all'interno dei kalathoi (Juan-Tresserras 2000).

\section{OLTRE I POTES MELEIROS: VASI PER LO STOCCAGGIO DI MIELE NELLE FONTI DO- CUMENTARIE}

Passata in rassegna la documentazione archeologica ed etnografica a supporto di un'interpretazione dei vasi a colletto etruschi come contenitori per miele, è necessario mettere in relazione questi dati con quanto attestato dalle fonti documentarie sui vasi destinati all'immagazzinamento dell'alimento.

L'uso della documentazione letteraria ed epigrafica per l'identificazione delle forme vascolari destinate a una specifica funzione non può essere distinta dai rilevanti problemi ermeneutici connessi all'individuazione dei nomi e della funzionalità dei vasi antichi sulla base delle fonti scritte (Lazzarini 1973-1974; Gulletta 1988). La pluralità degli usi che gli stessi contenitori potevano svolgere nell'antichità deve d'altronde esser sempre considerata, evitando di forzare con categorie concettuali moderne la documentazione antica.

È infatti particolarmente rischioso adeguare testimonianze molto eterogenee dal punto di vista cronologico (come i Deipnosofisti, i papiri documentari o le stesse iscrizioni) alla tassonomia archeologica, di norma focalizzata su dettagli poco percepiti in antichità o comunque non esplicitati al momento della stesura dei testi. Questi si soffermano sulla descrizione di elementi inconsueti: nell'ambito dell'apicoltura questo si riflette per esempio nella notevole cura con cui Columella descrive l'utensile in ceramica per affumicare le arnie, mentre si limita alla sola menzione dei vasa fictilia destinati a contenere il miele (Columella, Rust. 9. 15. 13).

\footnotetext{
${ }^{7}$ Per la presenza dei kalathoi in Italia: Bruni e Conde 1991 e in particolare Bencivenga Trillmich 1985 (Velia); Conde Berdós 1996 (Liguria e con bibliografia generale); Bruni 1992 (Etruria); Bertini 2004 (Populonia); Muscolino 2006 (Sicilia); Casini e Tizzoni 2010 (Italia settentrionale). In riferimento al contenuto dei kalathoi Tarradell e Sanmartí 1980: 315; Montanya Maluquer e Puig Ochoa 1979; più possibilisti a favore del miele Bonet Rosado e Mata Parreño 1997: 43-44; Bonet Rosado e Mata Parreño 2008, 156-158.
} 
Sebbene le fonti scritte facciano di frequente riferimento a vasi destinati a contenere miele, basti ricordare i papiri dell'archivio di Zenone di Cauno in età ellenistica e i testi romani sull'agricoltura, non sembrano essere individuabili riferimenti a peculiari tipi di vasi (Bortolin e Bruno 2006). Gli stessi vasa mellaria cui fa riferimento Plinio (HN 21. 82) devono essere con ogni probabilità considerati arnie, sulla scorta di un passo varroniano (Varro, Rust. 3. 16. 12: "melittona ita facere oportet, quos alii melitrophia appellant, eandem rem quidam mellaria" ed. C. Guiraud 1997). È invece Macrobio a riferire lo stesso termine (mellarium) a un contenitore destinato allo stoccaggio del miele (Macrob., Sat. 1. 12. 25).

Neppure la documentazione epigrafica consente di identificare forme destinate a questa specifica funzione, ma segnala soltanto la presenza della sostanza, ed eccezionalmente il peso. Nel mondo greco le anfore con graffito M / ME sono state interpretate come contenitori riusati per miele o vino mielato, corrispettivo del mulsum romano (Lawall 2000: 18-19). Neppure la piccola anfora da Siracusa con iscrizione verniciata (MEAIKPATA), destinata a contenere una bevanda di latte e miele mescolati, può essere considerata tout court un contenitore per miele (Biondi e Manganaro 2010: 58-61, Fig. 7; sulla bevanda Bortolin 2008: 24). Un grande vaso da Halos in Tessaglia presenta sì una chiara indicazione di contenuto graffita, ma viste le dimensioni non poteva essere destinato all'immagazzinamento di limitate quantità della sostanza o al suo trasporto (Reinders e Prummel 2003: 264, P257, app. 1, Fig. 6.3)

Le iscrizioni latine su vasi destinati a contenere miele sono attestate da poco più di una decina di esemplari (discussioni in Bortolin e Bruno 2006; Bortolin 2008: 175-180; Peña 2007: 103-105; Bassi 2008). Questi testi si trovano su vasi morfologicamente differenti: un'anforetta (Pompei, casa del Menandro, CIL IV, 9421; Allison 2006: 109, n. 588, tav. 43.1), un'olla biansata (Arcole, Bortolin e Bruno 2006), una forma kantharoide (Somma Lombardo, Olcese 1998: 202-203, tav. CXXXII), senza considerare la citata olla da Ventimiglia (Fig. 8). Le epigrafi sembrano essere incise post cocturam; realizzate quindi al momento dell'impiego per chiarire la materia trasportata o per precisarne la quantità ai fini di una transazione commerciale.

\section{CONSIDERAZIONI CONCLUSIVE}

Come si può mettere in relazione la documentazione archeologica fornita dalle olle a colletto e dai loro confronti etnografici con l'articolato quadro offerto dalle testimonianze letterarie ed epigrafiche sui vasi da miele?

In primo luogo è necessario aver chiaro il criterio della polifunzionalità dei contenitori antichi che consente di comprendere come, durante il loro ciclo di vita, questi manufatti possano presentare mutamenti di funzione determinati da scelte di economicità. Non bisogna poi dimenticare che gran parte dei contenitori era realizzata in materiale deperibile e pertanto risulta invisibile nella documentazione archeologica.

All'interno di un ambito funzionale ampio e poco caratterizzato, come quello dello stoccaggio e della movimentazione di una sostanza semi-solida, è indubbio l'uso di differenti tipi di recipienti sulla base della disponibilità domestica. Tale varietà è anche frutto della riparazione dei materiali e del loro continuo riuso, fenomeni determinanti per avere un quadro realistico della circolazione ceramica antica ${ }^{8}$. La documentazione epigrafica pervenuta 'fotografa' appunto questa situazione, dimostrando che alcuni contenitori (olle, anfore e anche forme aperte) sono stati utilizzati, in una fase della propria vita, per contenere miele. Questo invita quindi ad abbandonare una visione modernizzante, cioè una connessione univoca fra una forma vascolare precisa (olla a colletto) e una determinata funzione (conservazione del miele).

D'altra parte tale considerazione non deve dissuadere dal riscontrare anche in Etruria l'esistenza di una probabile forma vascolare specializzata (utile, ma non necessaria) per l'immagazzinamento del miele. La presenza ricorrente e standardizzata dell' attributo morfologico del colletto si può spiegare solo grazie a un'analogia etnografica che associa questa forma a un determinato uso 9 . I potes meleiros, connotati da un aspetto quasi identico e realizzati negli stessi anni in cui in Etruria venivano modellate le olle stamnoidi a colletto, costituiscono l'unico confronto morfologico per questi vasi etruschi.

Considerando poi la forte connessione fra il miele e talune pratiche funerarie (Gamurrini 1917; Sacco 1978: 81), l'eventuale motivazione ideologica a monte dell'uso di un vaso destinato a questa sostanza come

\footnotetext{
${ }^{8}$ Per una presentazione di questi fenomeni: Sullivan 1989 Mannoni e Giannichedda 1996: 148-167 e soprattutto Peña 2007. Due importanti casi studio (Corinto e Atene) sono discussi in Slane 2011 e Rotroff 2011.

9 L'etnografia è divenuta pratica comune sia per la comprensione del ciclo produttivo della ceramica antica (almeno a partire da Hampe e Winter 1962; Hampe e Winter 1965) sia per considerazioni formali e tipologiche, dove le classificazioni 'etnografiche' possono essere di particolare importanza per comprendere quelle 'archeologiche' (e.g. Piana Agostinetti 1988-1989: 674-688; di Gennaro e Depalmas 2011). Sugli assunti metodologici in riferimento all'analogia etnografica in archeologia Gallay 2011: 80-87.
} 


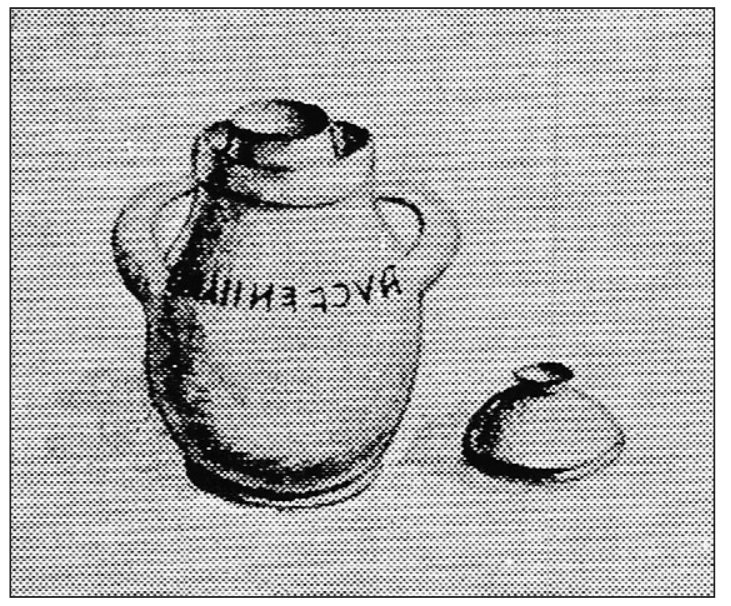

Figura 14. Olla stamnoide a colletto dalla Collezione Tommasi di Montaperti (da Cristofani 1979: Fig. 143/15).

cinerario potrebbe essere ben spiegata. Il miele era utilizzato nei riti di passaggio e connesso alla sfera ultraterrena: la scelta di un 'suo contenitore' potrebbe essere connessa a più profondi significati simbolici (Giuman 2014 e Baldoni 2013: 144-145, presentando arnie usate per sepolture infantili). Un esame dell'epigrafe sull'esemplare perduto dalla tomba degli Cvenle presso Montaperti (cat. 51 / Fig. 14) avrebbe potuto fornire preziose indicazioni a tal proposito, soprattutto se il testo fosse stato realizzato prima della cottura del vaso.

In conclusione, i confronti qui proposti, basati esclusivamente su analogie morfologiche, si prestano a fornire un'ipotesi di lavoro, con l'auspicio che analisi archeometriche possano rintracciare anche negli esemplari italiani tracce di miele ${ }^{10}$. Le stringenti affinità fra le olle stamnoidi a colletto e i potes meleiros iberici dovranno poi essere spiegate tenendo presenti le reti commerciali operanti nel Mediterraneo occidentale in età ellenistica e in particolare le interconnessioni esistenti fra Etruria e mondo iberico (Bruni e Conde 1991; Bruni 1992; Jolivet 2010 e in generale Gori 2006 e Della Fina 2007). Lo 'spostamento' di questa forma vascolare, valutato nel complesso della mobilità di uomini e merci lungo la via che fronteggiava la Francia del Sud, non sarà più un caso di 'migrazione tipologica', ma un fortunato prodotto di interazione culturale.

${ }^{10}$ Per l'identificazione delle tracce di miele: Notarstefano 2012, 109-113, 136-137 (cui si rimanda anche per un riepilogo sul dibattito in riferimento all'uso dell' archeometria per definire la funzionalità dei vasi). Per i casi iberici Oliveira e Morais 2014: 40, 44-45; Oliveira et alii 2014.

\section{BIBLIOGRAFIA}

Allison, P. M. (a cura di) 2006: The Insula of the Menander at Pompeii III: the Finds, a Contextual Study, Oxford.

Anderson-Stojanović, V. R. e Jones, J. E. 2002: “Ancient Beehives from Isthmia", Hesperia 71, 4, 345-376. http://dx.doi.org/10.2307/3182041

André, J. 1981: L'alimentation et la cuisine à Rome, $2^{\mathrm{a}}$ ed., Paris.

Arena, M. S. 1969: "Su alcuni frammenti di ceramica italo-megarese conservati nell' Antiquarium di Ostia”, Rivista di Studi Liguri XXXV, 101-121.

Balandier, C. 2004: "L'importance de la production du miel dans l'économie gréco-romaine", Pallas 64, 183-196.

Baldoni, D. 2013: "Riti, usi e corredi funerari a Iasos in epoca ellenistica", D. Baldoni, F. Berti, M. Giuman (eds.), Iasos e il suo territorio, Roma, 135-160.

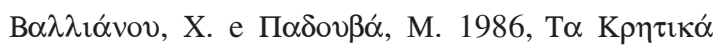

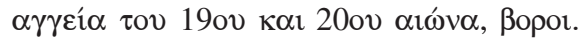

Barbieri, G. 2003: "Considerazioni sulla ceramica in uso a Norchia nel III secolo a.C. attraverso un corredo inedito da una tomba del fosso Pile", Rivista di Studi Liguri LXIX, 225-255.

Bassi, C. 2008: "Un contenitore per miele da Tridentum", P. Basso, A. Buonopane, A. Cavarzere e S. Pesavento Mattioli (eds.), Est enim ille flos Italiae... Vita economica e sociale nella Cisalpina romana, Verona, 287-294.

Bencivenga Trillmich, C. 1985: "La ceramica iberica da Velia. Contributo allo studio della diffusione della ceramica iberica in Italia", Madrider Mitteilungen 25, 20-33.

Bérato J. 2009: "Typologie diachronique et diffusion de la céramique modelée du Var, du $\mathrm{II}^{\mathrm{e}} \mathrm{s}$. av. J.-C. Au III ap. J.-C.", M. Pasqualini (ed.), Les céramiques communes d'Italie et de Narbonnaise. Structures de production, typologies et contextes inédits II ${ }^{e}$ s. av. J.-C. - III ap. J.-C., Napoli, 375-441.

Beretta, M. 2009: "Usi scientifici della cera nell' antichità", Quaderni Storici 130, 15-33.

Bertini, E. 2004: "Sombreros de Copa", M. L. Gualandi e C. Mascione (eds.), Materiali per Populonia 3, Firenze, 143-146.

Biondi, G. e Manganaro, G. 2010: "Relitti epigrafici per la storia del vino, di droghe e del miele nella Sicilia tardoellenistica", Epigraphica LXXII, 1-2, 51-67.

Bonet Rosado, H. e Mata Parreño, C. 1997: "The Archaeology of Beekeeping in Pre-Roman Iberia”, Journal of Mediterranean Archaeology 10, 1, 33-47. http://dx.doi.org/10.1558/jmea.v10i1.33 
Bonet Rosado, H. e Mata Parreño, C. 2008: "Las cerámicas ibéricas. Estado de la cuestión”, D. Bernal Casasola e A. Ribera i Lacomba (eds.), Cerámicas hispanorromanas. Un estado de la cuestión, Cádiz, 147-169.

Bormetti, M. 2014: "Api e miele nel Mediterraneo antico", Acme. Annali della Facoltà di Lettere e Filosofia dell'Università degli studi di Milano $67,1,7-50$.

Bortolin, R. 2008: Archeologia del miele, Mantova.

Bortolin, R. 2011: “Arnie, miele e api nella Grecia antica”, Rivista di Archeologia XXXV, 149-166.

Bortolin, R. e Bruno, B. 2006: "Il graffito 'melis' su un vaso di Arcole (VR). Considerazioni sui contenitori da miele nell'antichità", E. Bianchin Citton e M. Tirelli (eds.), ... ut... rosae ponerentur. Scritti di archeologia in ricordo di Giovanna Luisa Ravagnan, Treviso, 113-124.

Brentchaloff, D. 1980: "L'atelier du Pauvadou, une officine de potiers flaviens à Fréjus", Revue archéologique de Narbonnaise XIII, 73-114. http:// dx.doi.org/10.3406/ran.1980.1051

Bruni, S. 1992: "Presenze di ceramica iberica in Etruria", Rivista di Studi Liguri LVIII, 37-65.

Bruni, S. e Conde, M. J. 1991: "Presencia ibérica en Etruria y el mundo itálico a través de los hallazgos cerámicos de los siglos III-I a.C.”, J. Remesal e O. Musso (eds.), La presencia de material etrusco en la Península Ibérica, Barcelona, 543-576.

Burn, L. 1985: "Honey pots. Three white-ground cups by the Sotades Painter", Antike Kunst 28, 93-105.

Casini, S. e Tizzoni, M. 2010: "Kalathoi iberici e loro imitazioni nella Mediolanum celtica", Notizie Archeologiche Bergomensi 18, 165-178.

Cavagnaro Vanoni, L. 1986: "Aspetti inediti della necropoli del Calvario", Gli Etruschi di Tarquinia, Modena, 321-324, 349-350.

Cavagnaro Vanoni, L. 1996: Tombe tarquiniesi di età ellenistica. Catalogo di ventisei tombe a camera scoperte dalla Fondazione Lerici in località Calvario, Roma.

Cherici, A. 1989: "Granaî o arnie? Considerazioni su una classe fittile attica tra IX e VIII secolo a.C.", Atti dell'Accademia Nazionale dei Lincei. Classe di scienze morali, storiche e filologiche. Rendiconti 44, 215-230.

Chiesa, F. 2005: Tarquinia. Archeologia e prosopografia tra ellenismo e romanizzazione, Roma.

Cilliers, L. e Retief, F. P. 2008: "Bees, Honey and Health in Antiquity", Akroterion 53, 7-19. http:// dx.doi.org/10.7445/53-0-36

Colonna Di Paolo, E. e Colonna, G. 1978: Norchia I, Roma.
Conde Berdós, M. J. 1996: "La cerámica ibérica de Albintimilium y el tráfico mediterráneo en los siglos II-I a.C.", Rivista di Studi Liguri LXII, 115-168.

Crane, E. 1983: The Archaeology of Beekeeping, London.

Cristofani, M. (ed.) 1979: Siena: le origini. Testimonianze e miti archeologici, Firenze.

Cultrera, G. 1920: "Nuove scoperte nella necropoli tarquiniese", Notizie degli scavi di antichità. Accademia Nazionale dei Lincei 1920, 244-276.

Custer, A. 1928: "Lucca - Rinvenimenti archeologici a Ponte a Moriano", Notizie degli scavi di antichità. Accademia Nazionale dei Lincei 1928, 28-31.

D’Agata, A. L. e De Angelis, S. 2014: "Minoan beehives. Reconstructing the practice of beekeeping in bronze age Crete", Aegaeum 37, 349-357.

Del Chiaro, M. A. 1973: "An Iberian Sherd in Yougoslavia”, American Journal of Archaeology 77, 65-66. http://dx.doi.org/10.2307/503235

Delgado, M. 1996-1997: "Potes meleiros de Bracara Augusta", Portugália n.s. XVII-XVIII, 149-165.

Della Fina, G. M. (ed.) 2007: Etruschi, Greci, Fenici e Cartaginesi nel Mediterraneo centrale, Atti del XIV Convegno Internazionale di Studi sulla Storia e l'Archeologia dell'Etruria, Roma.

Di Gennaro, F e Depalmas, A. 2011: "Forni, teglie e piastre fittili per la cottura: aspetti formali e funzionali in contesti archeologici ed etnografici", F. Lugli, A. S. Stoppiello, S. Biagetti (eds.), Atti del $4^{\circ}$ Convegno Nazionale di Etnoarcheologia, Oxford, 56-61.

Dyson, S. L. 1976: Cosa: the Utilitarian Pottery, Memoirs of the American Academy in Rome XXXIIII, Roma.

Emiliozzi, A. 1974: La collezione Rossi Danielli nel Museo civico di Viterbo, Roma.

Esposito, A. M. (ed.) 1999: Principi Guerrieri. La necropoli etrusca di Casale Marittimo, Milano.

Ettlinger, E., Hedinger, B., Hoffmann, B., Kendrick, P. M., Pucci, G., Roth-Rubi, K., Schneider, G., von Schnurbein, S., Wells, C. M. e ZabehlickyScheffenegger, S. 1990: Conspectus formarum terrae sigillatae italico modo confectae, Bonn.

Fletcher Valls, D. 1953: "Una nueva forma en la cerámica ibérica de San Miguel de Liria (Valencia)", Zephyrus IV, 187-191.

Fortini, P. (a cura di) 1987: Monte Romano. Indagine di un territorio e materiali dell'Antiquarium, Roma.

Francis, J. E. 2012: "Experiments with an Old Ceramic Beehive", Oxford Journal of Archaeology 31, 143-159. http://dx.doi.org/10.1111/j.14680092.2012.00383.x 
Gabrieli, R. S. 2009: "Stability and Change in Ottoman Coarse Wares in Cyprus", The Annual of the American Schools of Oriental Research 64, 67-77.

Gallay, A. 2011: Pour une ethnoarchéologie théorique. Mérites et limites de l'analogie ethnographique, Paris.

Gambaro, L. 2006: "Morfologia ed uso di una forma ceramica a lunga continuità produttiva (II sec. a. C. - media età imperiale)", N. Cucuzza e M. Medri (a cura di), Archeologie. Studi in onore di Tiziano Mannoni, Bari, 383-387.

Gamurrini, G. F. 1917: "Di una iscrizione del territorio di Venosa", Atti dell'Accademia Nazionale dei Lincei. Classe di scienze morali, storiche e filologiche. Rendiconti s.5 XXVI, 98-102.

Gandolfi, D. e Gervasini, L. 2005: "La ceramica comune delle collezioni civiche del Museo Archeologico 'Girolamo Rossi' di Ventimiglia. Primo rapporto", SFECAG, Actes du congres de Vallauris, Marseille, 85-96.

Gandolfi, D. e Mennella, G. 2012: "Un vaso 'meleiro' con iscrizione graffita da Ventimiglia", G. Baratta e S. M. Marengo (eds.), Instrumenta Inscripta III. Manufatti iscritti e vita dei santuari in età romana, Macerata, 327-342.

Gargana, A. 1936: "Norchia (Vetralla) - Ritrovamento di tombe etrusche", Notizie degli scavi di antichità. Accademia Nazionale dei Lincei, 268-288.

Giuman, M. 2008: Melissa. Archeologia delle api e del miele nella Grecia antica, Roma.

Giuman, M. 2014: "Г $\lambda \alpha v \kappa о \varsigma ~ \pi \iota \omega v \mu \varepsilon \lambda \iota ~ \alpha v \varepsilon \sigma \tau \eta$. Ritualità e simbologia del miele nel mito di Glauco", I. Baglioni (ed.), Sulle rive dell'Acheronte. Costruzione e Percezione della Sfera del Post Mortem nel Mediterraneo antico, 2. L'antichità classica e cristiana, Roma, 75-87.

Gomes, M. V. 2007: "Vaso meleiro, de idade sidérica, dos Arrifes do Poço (Aljezur, Algarve)", Conimbriga 46, 73-88. http://dx.doi.org/10.14195/16478657_46_4

Gori, S. (ed.) 2006: Gli Etruschi da Genova ad Ampurias, atti del XXIV convegno di Studi Etruschi ed Italici, Pisa-Roma.

Gulletta, M. I. 1988: "Per un lessico greco dei vasi: storia degli studi e dei contributi dal 1828 al 1987", Annali della Scuola Normale Superiore di Pisa IIIs. 18, 1427-1439.

Hampe, R. e Winter, A. 1962: Bei Töpfern und Töpferinnen in Kreta, Messenien und Zypern, Mainz.

Hampe, R. e Winter, A. 1965: Bei Töpfern und Zieglern in Süditalien, Sizilien und Griechenland, Mainz.

Hayes, J. W. 1985: Etruscan and Italic Pottery in the Royal Ontario Museum. A Catalogue, Toronto.
Hayes, J. W. 2008: Roman Pottery. Fine-Ware Imports (The Athenian Agora XXXII), Princeton.

Holwerda, J. H. 1936: Het laat-grieksche en romeinsche gebruiksaardewerk uit het MiddellandscheZee-Gebied in het Rijksmuseum van Oudheden te Leiden, Leiden.

Jolivet, V. 1999: "De la prospection géophysique à la publication: tombes hellénistiques de Tarquinia", Journal of Roman Archaeology 12, 2, 476-482. http://dx.doi.org/10.1017/s1047759400018201

Jolivet, V. 2010: "Commerce, échanges, objets erratiques come marqueurs de rapports culturels?", Bollettino di Archeologia on line, 12-19.

Jones, J. E., Graham, A. J. e Sackett, H. 1973: “An Attic Country House below the Cave of Pan at Vari", The Annual of the British School at Athens 68, 355-443. http://dx.doi.org/10.1017/ s0068245400004524

Juan-Tresserras, J. 2000: "Estudio de contenidos en cerámicas ibéricas del Torrelló de Almazora (Castellón)", Archivo Español de Arqueología 73, 103-104. (Anexo del artículo Clausell, G., Izquierdo, I., Arasa, F. "La fase del Ibérico final en el asentamiento del Torrelló del Boverot (Almazora, Castellón): dos piezas cerámicas singulares". http://dx-doi.org/10.3989/aespa.2000. v73.319).

Kardara, C. 1961: "Dyeing and Weaving Works at Isthmia", American Journal of Archaeology 65, 261-266. http://dx.doi.org/10.2307/501686

Kirsopp Lake, A. 1934-1935: “Campana Suppellex (The Pottery Deposit at Minturnae)", Bollettino dell'Associazione internazionale degli Studi Mediterranei $\mathrm{V}, 4-5,97-114$.

Lafaye, G. 1904: "Mel”, Daremberg-Saglio III, 2, 1701-1706.

Lawall, M. L. 2000: "Graffiti, Wine Selling, and the Reuse of Amphoras in the Athenian Agora, ca. 430 to 400 B.C.", Hesperia 69, 1, 3-90. http:// dx.doi.org/10.2307/148365

Lawall, M. L., Papadopoulos, J. K., Lynch, K. M., Tsakirgis, B., Rotroff, S. I. e MacKay, C. 2001: "Notes from the Tins: Research in the Stoa of Attalos, Summer 1999", Hesperia 70, 2, 163-182. http://dx.doi.org/10.2307/2668481

Lazzarini, M. L. 1973-1974: "I nomi dei vasi greci nelle iscrizioni dei vasi stessi", Archeologia Classica 25-26, 341-375.

Mannoni, T. e Giannichedda, E. 1996, Archeologia della produzione, Torino.

Martelli, M. 1976: "Recensione a Emiliozzi 1974", Prospettiva 4, 42-49.

Martelli, M. 1981: "Populonia: cultura locale e contatti con il mondo greco", L'Etruria mineraria, 
atti del XII Convegno di Studi Etruschi e Italici, Firenze, 399-427.

Massoul, M. 1934: Corpus Vasorum Antiquorum, France. Musée National de Sèvres, Paris.

Mata Parrenño, C. e Bonet Rosado, H. 1992: "La cerámica ibérica. Ensayo de tipología", Estudios de Arqueología Ibérica y Romana. Homenaje a Enrique Pla Ballestrer, Valencia, 117-174.

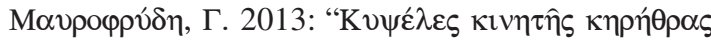

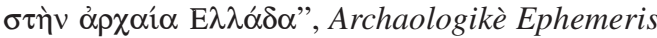
$152,15-27$.

Milanese, M. e Mannoni, T. 1984: "Gli Etruschi a Genova e il commercio mediterraneo", Studi Etruschi LII, 117-146.

Montanya Maluquer, R. e Puig Ochoa, M. R. 1979: "La cerámica ibérica pintada tardía y sus perduraciones", Rivista di Studi Liguri XLV, 221-230.

Morais, R. 2006: "Potes meleiros e colmeias em cerâmica: uma tradição milenar", Saguntum 38, 149-161.

Morais, R. 2014a: "Notícia sobre vaso grego destinado ao transporte e conservação de mel", Portugália n.s. XXXV, 95-100.

Morais, R. 2014b: "News about a Greek vase used to transport and conserve honey", P. Bádenas de la Peña et alii (eds.) Per speculum in aenigmate. Miradas sobre la antigüedad. Homenaje a Ricardo Olmos, Madrid, 256-258.

Morel, Ch. 1877: “Apes”, Daremberg-Saglio I, 1, 304-305

Moretti, M. e Sgubini Moretti, A. M. (eds.) 1983: I Curunas di Tuscania, Roma.

Morín de Pablos, J. e de Almeida, R. R. 2014: "La apicultura en la Hispania romana: producción, consumo y circulación”, M. Bustamante Álvarez e D. Bernal Casasola (eds.) Artifices Idoneos. Artesanos, talleres y manufacturas en Hispania, Anejos de Archivo Español de Arqueología LXXI, Mérida, 269-294.

Muscolino, F. 2006: "Kalathoi iberici da Taormina. Aggiornamento sulla diffusione della ceramica iberica dipinta in Sicilia", Archivo Español de Arqueología 79, 217-224. http://dx.doi.org/10.3989/ aespa.2006.v79.11

Nielsen, E. R. 1993: "Honey in Medicine", Sesto congresso internazionale di Egittologia, Torino, 415-419.

Notarstefano, F. 2012: Ceramica e alimentazione. L'analisi chimica dei residui organici nelle ceramiche applicata ai contesti archeologici, Bari.

Olcese, G. 1993: Le ceramiche comuni di Albintimilium. Indagine archeologica e archeometrica sui materiali dell'area del Cardine, Firenze.

Olcese, G. (ed.) 1998: Ceramiche in Lombardia tra II a.C. e VII d.C. Raccolta dei dati editi, Mantova.
Oliveira, C. e Morais, R. 2014: "Estudos de cromatografia aplicados à arqueologia romana: apresentação de resultados preliminares", Ciências $e$ técnicas do Património. Revista da Faculdade de Letras XIII, 37-60.

Oliveira, C., Morais, R., Araújo, A., KuzniarskaBiernacka, I., Parpot, P., Neves, I. C. e Fonseca, A. M. 2014: "Análise de fragmentos cerâmicos de potes meleiros e colmeias por cromatografia gasosa acoplada à espectroscopia de massa”, R. Morais, A. Fernández e M. J. Sousa (eds.), As produções cerámicas de imitação na Hispania, II Congresso Internacional da SECAH - EX OFFICINA HISPANA, Braga, 599-610.

Peignard-Giros A. 2012: "Les céramiques communes à Délos à l'époque hellénistique tardive (II ${ }^{\mathrm{e}} \mathrm{I}^{\mathrm{er}}$ siècles av. J.-C.)", C. Batigne Vallet (ed.) Les céramiques communes dans leur contexte régional. Faciès de consommation et mode d'approvisionnement, Lyon, 243-256.

Peña, J. T. 2007: Roman Pottery in the Archaeological Record, Cambridge.

Peroni, R. 1998: "Classificazione tipologica, seriazione cronologica, distribuzione geografica", Aquileia Nostra LXIX, 10-27.

Piana Agostinetti, P. (ed.) 1988-1989: "Il concetto di tipo: un'esperienza nel campo della ceramica", Origini 14, 653-695.

Puppo, P. 1995: Le coppe megaresi in Italia, Roma. Regert, M., Colinart, S., Degrand, L. e Decavallas, O. 2001: "Chemical Alteration and Use of Beeswax through Time. Accelerated Ageing Tests and Analysis of Archaeological Samples from Various Environmental Contexts", Archaeometry 43, 4, 549-569. http://dx.doi.org/10.1111/1475-4754.00036

Reinders, H. R. e Prummel, W. (eds.) 2003: Housing in New Halos. A Hellenistic Town in Thessaly, Greece, Lisse-Abingdon-Exton-Tokyo.

Rivet, L. 1982: "La céramique culinaire micacée de la région de Fréjus (Var)", Revue archéologique de Narbonnaise XV, 1, 243-262. http://dx.doi. org/10.3406/ran.1982.1208

Romualdi, A. 1985: "Il popolamento in età ellenistica a Populonia: le necropoli", G. Camporeale (ed.), L'Etruria mineraria, Milano, 185-218.

Rotroff, S. 2006: Hellenistic Pottery. The Plain Wares, The Athenian Agora XXXIII, Princeton.

Rotroff, S. 2011: "Mended in Antiquity: Repairs to Ceramics at the Athenian Agora", M. Lawall e J. Lund (eds.), Pottery in the Archaeological Record: Greece and Beyond, Aarhus, 117-134.

Sacco, G. 1978: "Due note epigrafiche. 2. Il miele e la cera nelle iscrizioni funerarie", Rivista di Filologia e d'Istruzione Classica 106, 1, 77-81. 
Santrot, M-H. e Santrot, J. 1995: La citerne 5 et son mobilier, Bolsena VII, Rome.

Serra, F. R. 1970: "La ceramica fine con tracce di decorazione dipinta etc.", Notizie degli scavi di antichità. Accademia Nazionale dei Lincei, II suppl., II, 504-552.

Serra Ridgway, F. R. 1986: "b) La necropoli del Fondo Scataglini (Villa Tarantola)", M. Bonghi Jovino (ed.), Gli Etruschi di Tarquinia, 324-340, 350-353.

Serra Ridgway, F. R. 1996: I corredi del fondo Scataglini a Tarquinia. Scavi della Fondazione Ing. Carlo M. Lerici del Politecnico di Milano per la Soprintendenza archeologica dell'Etruria meridionale, Milano.

Shepherd, E. J. 1992: "Ceramica acroma, verniciata ed argentata", A. Romualdi (ed.), Populonia in età ellenistica. I materiali dalle necropoli, atti del seminario, Firenze, 152-178.

Slane, K. W. 2011, "Repair and Recycling in Corinth and the Archaeological Record", M. Lawall e J.
Lund (eds.) Pottery in the Archaeological Record: Greece and Beyond, Aarhus, 95-106.

Sullivan, A. P. 1989: "The Technology of Ceramic Reuse: Formation Processes and Archaeological Evidence", World Archaeology 21, 1, 101-114. http://dx.doi.org/10.1080/00438243.1989.998 0093

Tarradell, M. e Sanmartí, E. 1980 : “L’état actuel des études sur la céramique ibérique", Céramiques Hellénistiques et Romaines, Besançon, 303-330.

Van Ingen, W. 1933, Corpus Vasorum Antiquorum. United States of America, University of Michigan, Fascicule 1, Cambridge (Mss.).

Vázquez Hoys, A. M. 1991: "La miel, alimento de eternidad", Alimenta. Estudios en homenaje al Dr. Michel Ponsich, Gerión Anejos III, 61-93.

Recibido: 20-01-2015

Aceptado: 09-04-2015 